\title{
A quasi-randomised, controlled, feasibility trial of GLITtER (Green Light Imaging Interpretation to Enhance Recovery) - a psychoeducational intervention for adults with low back pain attending secondary care
}

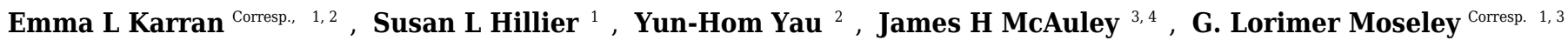 \\ ${ }^{1}$ School of Health Sciences, University of South Australia, Adelaide, South Australia, Australia \\ 2 Royal Adelaide Hospital, Adelaide, South Australia, Australia \\ 3 Neuroscience Research Australia, Sydney, New South Wales, Australia \\ 4 School of Medical Sciences, Faculty of Medicine, University of New South Wales, Sydney, New South Wales, Australia \\ Corresponding Authors: Emma L Karran, G. Lorimer Moseley \\ Email address: emma.karran@mymail.unisa.edu.au, Lorimer.Moseley@gmail.com
}

Background: Although it is broadly accepted that clinicians should endeavour to reassure patients with low back pain, to do so can present a significant clinical challenge. Guidance for how to provide effective reassurance is scarce and there may be a need to counter patient concerns arising from misinterpretation of spinal imaging findings. 'GLITtER' (Green Light Imaging Intervention to Enhance Recovery) was developed as a standardised method of communicating radiological findings in a manner that is reassuring and promotes engagement in an active recovery. This feasibility study is an important step towards definitive testing of its effect.

Methods: This feasibility study was a prospective, quasi-randomised, parallel trial with longitudinal follow-up, involving sampling of patients attending a spinal outpatient clinic at a metropolitan hospital. English speaking adults (18-75 years) presenting to the clinic with low back pain and prior spinal imaging were considered for inclusion. Eligible patients were allocated to receive a GLITtER consultation or a standard consultation (as determined by appointment scheduling and clinician availability), and were blinded to their allocation. Full details of the GLITtER intervention are described in accordance with the Tidier template. Follow-up data were collected after 1 and 3 months. The primary outcome of this study was the fulfillment of specific feasibility criteria which were established a priori. Determination of a sample size for a definitive randomised controlled trial was a secondary objective.

Results: 276 patients underwent preliminary screening and 31 patients met the final eligibility criteria for study inclusion. 17 participants were allocated to the intervention group and 14 were allocated to the control group. 3 month follow-up data were available from $42 \%$ of the 31 enrolled participants $(\mathrm{N}=13,6$ intervention, 7 control). Feasibility indicators for consent, resource burden and acceptability of the GLITtER intervention were met, however participant recruitment was slower than anticipated and an acceptable follow-up rate was not achieved.

Conclusions: Failure to achieve pre-specified recruitment and follow-up rates were important outcomes of this feasibility study. We attribute failure to issues that are likely to be relevant for other clinical trials with this population. It is realistic to consider that these challenges can be overcome through careful strategy, ample funding and continued partnership with health care providers.

Trial registration: The trial was registered on the Australian and New Zealand Clinical Trials Registry 
on 28/2/2017 (ACTRN12617000317392). 
8 A quasi-randomised, controlled, feasibility trial of GLITtER (Green Light Imaging

9 Interpretation to Enhance Recovery) - a psychoeducational intervention for

10 adults with low back pain attending secondary care

Emma L. Karran ${ }^{1,2}$, Susan L. Hillier ${ }^{1}$, Yun-Hom Yau², James H. McAuley ${ }^{3,4}$, G. Lorimer Moseley ${ }^{1,3}$

${ }^{1}$ School of Health Sciences, University of South Australia, Adelaide, SA; ${ }^{2}$ Royal Adelaide Hospital, Adelaide, SA;

${ }^{3}$ Neuroscience Research Australia, Sydney, NSW; ${ }^{4}$ School of Medical Sciences, Faculty of Medicine, University of

15 New South Wales, Sydney, NSW

17 Corresponding author:

G. Lorimer Moseley 


\section{Abstract}

Background: Although it is broadly accepted that clinicians should endeavour to reassure patients with low back pain, to do so can present a significant clinical challenge. Guidance for how to provide effective reassurance is scarce and there may be a need to counter patient concerns arising from misinterpretation of spinal imaging findings. 'GLITtER' (Green Light Imaging Intervention to Enhance Recovery) was developed as a standardised method of communicating radiological findings in a manner that is reassuring and promotes engagement in an active recovery. This feasibility study is an important step towards definitive testing of its effect.

Methods: This feasibility study was a prospective, quasi-randomised, parallel trial with longitudinal follow-up, involving sampling of patients attending a spinal outpatient clinic at a metropolitan hospital. English speaking adults (18-75 years) presenting to the clinic with low back pain and prior spinal imaging were considered for inclusion. Eligible patients were allocated to receive a GLITtER consultation or a standard consultation (as determined by appointment scheduling and clinician availability), and were blinded to their allocation. Full details of the GLITtER intervention are described in accordance with the TIDieR template. Follow-up data were collected after 1 and 3 months. The primary outcome of this study was the fulfillment of specific feasibility criteria which were established a priori. Determination of a sample size for a definitive randomised controlled trial was a secondary objective.

Results: 276 patients underwent preliminary screening and 31 patients met the final eligibility criteria for study inclusion. 17 participants were allocated to the intervention group and 14 were allocated to the control group. 3 month follow-up data were available from $42 \%$ of the 31 enrolled participants $(\mathrm{N}=$ 13, 6 intervention, 7 control). Feasibility indicators for consent, resource burden and acceptability of the GLITtER intervention were met, however participant recruitment was slower than anticipated and an acceptable follow-up rate was not achieved. Based on analysis of our pre-specified feasibility criteria, proceeding with a full-scale RCT is not recommended without process revision.

Conclusions: Failure to achieve pre-specified recruitment and follow-up rates were important outcomes of this feasibility study. We attribute failure to issues that are likely to be relevant for other clinical trials with this population. It is realistic to consider that these challenges can be overcome through careful strategy, ample funding and continued partnership with health care providers.

Trial registration: The trial was registered on the Australian and New Zealand Clinical Trials Registry on 28/2/2017 (ACTRN12617000317392). 


\section{Introduction}

58 Patients with low back pain (LBP) attending secondary care settings can be characterised by high pain 59 intensity, poor function and higher frequency of poor prognosis than patients attending primary care 60 (Karran et al. 2017b; Mors $\varnothing$ et al. 2013; Mors $\varnothing$ et al. 2014). Consequently, their contribution to the massive burden of LBP (Vos et al. 2016) is likely to be significant. General practitioners (GPs) frequently refer their patients to specialist secondary care clinics for surgical opinion ((ABS) 2015; Coulter 1998) however surgery is recommended for only a small proportion of cases (Li \& Yen 2010; Robarts et al. 2017). For the remainder, clinicians are generally challenged to provide time-efficient, guideline-based, conservative care recommendations. Screening tools have been used to determine risk and optimal pathways for back pain patients in primary care, but systematic review data (Karran et al. 2017a) are underwhelming and they seem to offer little benefit within the context of secondary care (Karran et al. 68 2017b).

There is widespread current agreement in LBP clinical practice guidelines that clinicians should deliver high quality information as a key component of their management (O'Connell et al. 2016; Wong et al. 2017). In particular, patients should be reassured that their condition is not likely to be serious, that a favourable outcome is usual and that activity levels should be resumed as soon as possible ((NICE) 2016; Koes et al. 2010; Qaseem et al. 2017; Stochkendahl et al. 2017). While patient reassurance can be considered a central tenet of this approach, practical guidance for how to effectively deliver reassurance in the clinical setting is lacking (Hasenbring \& Pincus 2015; Traeger et al. 2015). Furthermore, the impact of providing reassuring information on pragmatic outcomes appears to be poorly understood

78 (Pincus \& McCracken 2013; Traeger et al. 2017).

80 Counter to clinicians' efforts to reassure, the communication of spinal imaging findings have been 81 suggested to have the potential to increase patients' fear of re-injury and reduce their likelihood of a good outcome (Roland \& van Tulder 1998). Adverse effects of early imaging of the lumbar spine have also been reported (Graves et al. 2012; Webster \& Cifuentes 2010). That most LBP patients attending secondary care present with spinal images (Sears et al. 2016) and that these images are routinely considered during the consultation, provides opportunity for careful consideration of how imaging results are interpreted and communicated in this setting. 
88 Recent evidence reveals a high prevalence of common degenerative features in the imaging reports of asymptomatic adults (Brinjikji et al. 2014). This indicates that many of these features - particularly when found incidentally - should not be considered pathological and instead be regarded as normal age-related changes. Coherent with this interpretation is that degenerative findings have been found to be poorly associated with current pain (Brinjikji et al. 2014; Carragee et al. 2006; Suri et al. 2014) and prognosis (Carragee et al. 2005; De Schepper et al. 2016; Jensen et al. 2014; Steffens et al. 2014). Considering this evidence, we developed and tested an intervention framework based on using imaging findings for clinical benefit (Karran et al. In press (accepted 9 November 2017)). This psycho-educational intervention, referred to as 'GLITtER' (Green Light Imaging Intervention to Enhance Recovery), involves a standardised method of communicating radiological findings in a manner designed to reassure patients and promote engagement in an active recovery.

A feasibility trial, conducted in the spinal outpatient setting at an Australian metropolitan hospital, is the crucial first step towards definitive testing of this intervention. The primary aim of this study was to determine the feasibility of recruitment and retention, assessment procedures, implementation and acceptability of the GLITtER intervention for LBP patients attending a spinal outpatient clinic. Secondary objectives included the identification of modifications needed in the design of a larger effectiveness trial and provision of data to enable calculation of an appropriately powered sample for a subsequent effectiveness trial.

\section{Methods}

\section{Trial approval, registration and reporting}

111 The Research Ethics Committees at the Royal Adelaide Hospital (protocol no. 150308) and the University 112 of South Australia (protocol no. 0000034887 ) provided approval for this study. The full protocol was 113 pre-registered on the Open Science Framework (https://osf.io/8zrq3/) and the trial was registered on 114 the Australian and New Zealand Clinical Trials Registry (www.anzctr.org.au, registration number:

115 ACTRN12617000317392). Trial registration included details of all items from the World Health 116 Organisation Trial Registration Data Set (see Appendix 1). This study has been reported according to the 117 CONSORT 2010 guideline for transparent and quality reporting of randomised pilot and feasibility trials 118 (www.consort-statement.org) (see Appendix 2). 


\section{Study Design}

121 This investigation was a prospective, quasi-randomised feasibility trial with longitudinal follow-up,

122 involving sampling of patients attending the Spinal Assessment Clinic (SAC). The study also adopted an

123 adaptive trial design, whereby modifications could be made during its conduct with the purpose of

124 increasing the probability of success of the study procedure or the intervention. Adaptations were

125 made during recruitment of the first one-third of participants, as pre-specified in the protocol

126 (https://osf.io/8zrq3/).

127

\section{Study setting and participants}

129 The study was conducted in the SAC, which operates in the Spinal Outpatient Department at a large 130 metropolitan hospital in South Australia. The SAC is a Physiotherapist-led clinic attended by patients 131 who warrant non-urgent consultation, as identified by a paper-based triage procedure prior to 132 appointment scheduling. All patients aged between 18 and 75 years who were scheduled to attend the

133 SAC with a lumbar spine disorder were considered for inclusion. To be eligible, patients were required to 134 be able to speak and understand (verbal and written) English and have access to recent images of their 135 lumbar spine. Baseline pain duration was not an eligibility consideration, however it was anticipated 136 that patients presenting to the SAC were likely to have experienced pain for longer than 3 months, 137 based on usual minimum timeframes for appointment scheduling. Patients with a history of lumbar 138 spine surgery were excluded.

Intervention

141 Participants allocated to the intervention group received a 'GLITtER' consultation, integrated into the

142 standard SAC consultation. Participants allocated to the control group received a 'standard

143 consultation'. Full details of the consultations are provided in Table 1 in accordance with the TIDieR

144 template for intervention description and replication (see www.equator-network.org). In brief - a

145 routine, or 'standard' consultation involves comprehensive patient assessment, review and

146 interpretation of relevant investigations and discussion of management recommendations. A key

147 objective of the patient-centered interaction is identification of potential surgical candidates, or the 148 guidance of patients appropriate for continued conservative care towards community-based options.

149 The GLITtER consultation includes all components of the standard consultation but is enhanced by the 150 implementation of a standardised framework through which imaging findings are interpreted (designed 
151 to optimise patient reassurance), includes provision of take-home resource, emphasizes the need for an 152 active recovery, and offers links to further information.

153

\section{Staff Training}

155 Two SAC clinicians (clinicians 1 and 2) volunteered to deliver the GLITtER intervention and were 156 provided with training. Two clinicians (clinicians 3 and 4) remained naïve to GLITtER and agreed to 157 deliver the control intervention. A 3-stage training process was conducted for clinicians 1 and 2 . The 158 initial stage involved a meeting to provide background, rationale and an overview of the planned 159 intervention. Within this session, a neurosurgeon (YHY) led instruction and discussion of the imaging 160 interpretation strategy developed for GLITtER. The second stage involved providing clinicians 1 and 2 161 with the framework for the GLITtER intervention, with instruction to undertake self-directed 162 familiarization with the content (see Appendix 3). The third stage was a face-to-face session with the 163 principal researcher to discuss the intervention framework and practice strategies for clinical 164 implementation. The total time for training and familiarisation was 2-2.5 hours.

\section{Outcomes}

The primary outcome of this study was the fulfillment of specific feasibility criteria that were established a-priori (see Table 2) and designed to demonstrate that a subsequent effectiveness trial would be likely to be acceptable (to both patients and clinicians) and have sound methods and procedures likely to lead to successful trial completion. These criteria were considered and reported under the domains of process, resource and scientific considerations (Thabane et al. 2010) and acceptability. Process considerations referred to steps essential for the success of a larger study including rates of participant recruitment and retention. Resource considerations related to time and budget issues and included participant and clinician burden. Scientific considerations included estimates of treatment effect, variance of effect and data acquisition for a subsequent sample size calculation. We classified criteria that met the standards specified in Table 2 as "achieved" and criteria that failed to meet these prespecified standards (and would need addressing or adapting before proceeding with further testing of the intervention as "review".

The secondary outcomes of this study were:

181 1. Attainment of data permitting calculation of an appropriately powered sample for a subsequent RCT (i.e. the standard deviation of the NRS score for pain at 3-month follow-up). 
183 2. Exploratory analysis of between-group differences for changes in pain, disability, and kinaesiophobia 184 (from baseline to 3 month follow-up).

185 3. Identification of ceiling or floor effects.

186 (These outcomes were considered and reported under the domain of scientific considerations.)

187

188 Sample Size

189 We aimed to allocate 40 study participants to intervention or control groups using a quasi-randomised 190 procedure. This sample size was considered adequate for a feasibility study (designed principally to 191 assess feasibility of recruitment and procedures), and sufficient to inform a power analysis for a 192 subsequent randomised controlled trial (Hertzog 2008).

193

194

Procedure

195 The full study procedure, including preliminary and primary screening procedures to determine patient 196 eligibility for participation, is outlined in Figure 1.

197

198

Confirmation of participant eligibility

199 At completion of the SAC assessment, all clinicians completed a 5-item checklist to confirm final 200 eligibility for participation in this study. The intention of this checklist was to exclude patients who 201 required subsequent investigations, interventions or surgical intervention, or patients who had 202 pathology requiring significant caution with activity. It also confirmed that patients had functional or 203 exercise restrictions, such that an intervention to promote activity was warranted. Participants meeting

204 all criteria were notified of their inclusion in the study and were informed regarding subsequent follow205 up procedures.

206

207 Group allocation and participant blinding

208 Participants were allocated to the GLITtER Intervention or the control group via a quasi-randomised 209 procedure devised to cause minimal interference to clinic processes and also allow between-group 210 comparisons. Group allocation was determined by appointment scheduling and clinician availability, 211 such that patients were seen in order of their arrival at the clinic by the first available clinician. Patients 212 meeting the primary eligibility criteria were thereby pragmatically allocated to the intervention or 213 control conditions. Clinicians 1 and 2 were advised to provide no more than one GLITtER intervention 
214 per clinic session to minimise potential time burden and disruption to clinic flow. Study participants

215 were unaware whether they received a GLITtER consultation or a standard consultation.

216

217 Data Collection

218 Baseline demographic and outcome data were hand-recorded by participants on purpose-designed

219 forms. Follow-up outcome data were obtained via completion of postal questionnaires. All data were

220 entered onto a password protected excel spreadsheet. Participants were requested to complete the

221 Recommended Minimum Data Set for LBP Research (Deyo et al. 2014) and the Tampa Scale for

222 Kinaesiophobia-11 (TSK-11) (Tkachuk \& Harris 2012) at study inception. At 1 month follow-up,

223 participants were mailed numeric rating scales (NRS) for pain and disability, 3 questions about

224 reassurance (Appendix 4), and a participant experience questionnaire (Appendix 5). At 3 month follow-

225 up participants were requested to complete pain and disability NRS, the Roland Morris Disability

226 Questionnaire (RMDQ)(Roland \& Morris 1983), and the TSK-11. Health care utilisation was also

227 evaluated at 1 and 3 months (Appendix 5).

228

\section{Interim study evaluation}

230 Interim evaluation of the trial was undertaken after $30 \%$ of participants had been recruited into the 231 study. We considered problems with participant recruitment and discussed any clinician concerns or 232 suggestions. We also reviewed participant responses for completeness and reports of intervention 233 acceptability. Study protocol modifications were considered and implemented as considered 234 appropriate by the research team, and all changes were recorded.

\section{Statistical Methods}

237 Baseline clinical and demographic characteristics of the participants were reported using descriptive 238 statistics. Patient eligibility, recruitment and retention rates were calculated, and reasons for refused 239 consent were recorded where they were available. Questionnaire completion rates were also

240 calculated. The standard deviation of the primary outcome measure to be used in a future effectiveness

241 trial (pain NRS at 3 months) was used to inform the sample size calculation for a larger RCT. We planned

242 to conduct exploratory analysis of between-group differences in change scores for pain, disability and

243 kinaesiophobia (from baseline to 3-month follow-up) using ANOVA, acknowledging that estimates of

244 treatment effect should be assumed to be imprecise due to the non-randomised allocation procedure 245 and small sample size. 
Results

\section{Baseline characteristics of participants}

25031 patients were enrolled in this study between the $2^{\text {nd }}$ March and the $14^{\text {th }}$ July, 2017 , with the trial ceased due to temporary closure of the service. Detailed baseline data were collected for all participants consistent with the Recommended Minimum Dataset for LBP research (Deyo et al. 2014) (see Appendix 6). 63\% of study participants were female, and the mean age of participants was 50.1 years (standard deviation (SD) 14.0, range 20-75 years). All participants reported experiencing LBP for more than 6 months. Key baseline data are reported in Table 3.

256

257

258

259

260

261

262

263

264

265

266

267

268

269

270

271

272

273

274 275

276

\section{Interim evaluation}

Interim evaluation occurred after the first 12 participants had been recruited into the trial. The main issue discussed was the failure to consistently meet the weekly target for participant recruitment. An unforeseen change to clinic process (with the result that fewer patients who were likely to be recommended for conservative management received SAC appointments) was identified as the reason for the lower number of patients meeting the final eligibility criteria than anticipated. While no significant resolution to this issue was identified, the exclusion of patients who reported a past history of spinal surgery was discussed. We recognised that many of these patients did not require further imaging or surgical opinion and had the potential to benefit from GLITtER. The primary eligibility criteria were revised to permit inclusion of participants who had had no more than 1 surgical procedure (more than 2 years prior) and who met all other inclusion criteria.

At this interim stage, follow-up data were available from 5 participants - 3 of whom received a GLITtER Consultation. $100 \%$ of responses on the Participant Experience Questionnaires were favourable (i.e. responded to the participant satisfaction statements with ratings of "agree" or "strongly agree"). Clinicians delivering the GLITtER intervention also reported being satisfied with study processes. They perceived the GLITtER intervention to be well accepted by patients and felt confident in their ability to deliver it time efficiently and with competence. No specific concerns were raised.

\section{Achievement of feasibility criteria}


277 Achievement outcomes of the pre-specified feasibility are provided in Table 2 and detailed below.

278

279 Process considerations

280 A total of 31 participants were recruited from 15 clinic sessions. A CONSORT flow diagram detailing 281 study recruitment is provided in Figure 2. Of the 101 patients who met the primary eligibility criteria, 28224 patients declined involvement. This resulted in a consent rate of $75 \%$, which was considered 283 acceptable according to our pre-specified criteria. In addition to this however, $8 \%$ did not complete 284 signed consent forms due to unspecified reasons. An average of 2 participants were recruited each 285 clinic session, which was much less than our target recruitment rate of 4 participants per clinic session. 286 Further consideration and revision of recruitment feasibility is required. Acceptable participant follow287 up response rates were also not achieved, with 1 and 3-month outcome data available for $36 \%$ and $42 \%$ 288 of participants respectively.

289

290

\section{Resource considerations}

291 Participants completed $95 \%$ of all data items at baseline. $96 \%$ of items on the Recommended Minimum 292 Dataset were completed and 92\% of items on the TSK-11 were completed. Clinician burden was 293 evaluated by the duration of the GLITtER consultations in comparison with standard consultations. 54 294 SAC consultations (11 GLITtER consultations and 43 Standard Consultations) were timed by clinicians 1 and 2. The mean duration of the GLITtER consultations was 41 minutes $(S D=7$, minimum 30, maximum 50). The mean duration of standard consultations was 40 minutes ( $S D=9$ minutes, minimum 25 , maximum 70). 3 of the standard consultations exceeded 50 minutes. If these consultations were excluded, the mean duration of the Standard Consultations was reduced to 39 minutes $(S D=7)$.

\section{Management considerations}

$100 \%$ of responses on the participant experience questionnaires (for participants who received a GLITtER consultation) were favourable (i.e. participants responded to the satisfaction statements with ratings of "agree" or "strongly agree"). Clinicians 1 and 2 both reported that they were "confident" that they could integrate GLITtER into standard practice on an ongoing basis (on a 4-point Likert scale ranging from "not at all confident" to "very confident"). One clinician was "very confident" and the other was "confident" that integrating GLITtER into routine consultations would enhance SAC care. 
309 Based on a linear mixed effects model with two time points (baseline and 3 months), power $=80 \%$, Type

3101 error $=5 \%$, an expected correlation between baseline and 3 months measurements of 0.5 , a MCID of 1 , 311 and a SD of 2.1, then 53 patients are required per group. Allowing for $15 \%$ loss to follow-up, a sample 312 size of 63 participants per group is required for an appropriately powered RCT.

313

314 Mean pain, disability, and kinaesiophobia scores at baseline and 3 month follow-up are provided in

315 Table 4. We did not calculate change scores or conduct exploratory analysis of between-group 316 differences (as specified a priori) due to the small sample sizes achieved in each group. No ceiling or 317 floor effects were observed.

318

319

320

Discussion

321

322

This investigation has highlighted some important operational challenges impacting the feasibility of 323 successfully conducting a future effectiveness trial of GLITtER (Green Light Imaging Interpretation to Enhance Recovery). While pre-specified targets for consent rates, resource burden and acceptability of the GLITtER intervention were met, participant recruitment was slower than anticipated and an acceptable follow-up rate was not achieved.

327

Recruitment of participants into the study occurred at half of the anticipated rate, due largely to fewer patients meeting the final eligibility criteria than expected. An identified contributor to this was the change to referral management processes such that patients who were unlikely to be candidates for intervention were no longer offered SAC appointments. (Instead, these patients and their General Practitioners were informed via a letter that community-based management was most appropriate.) Although the number of patients attending the SAC who were eligible for this study was low, this may not indicate that the number of individuals suitable for the GLITtER intervention is also low. Instead, it may indicate that there is need to further consider how best to access the patients for whom GLITtER may offer potential benefit. Alternatively, plans to further investigate the GLITtER intervention in the spinal outpatient setting must carefully consider patient triage procedures, allow for slower recruitment, and investigate the potential for recruitment at multiple sites. The wording on the information sheet should also be reconsidered to avoid the risk that some patients may have declined participation out of 
340 concern that they would be given reassuring information during their consultation, rather than the

341 information that they were seeking.

342

343 We achieved a 3-month participant follow-up rate of only $42 \%$, which failed to meet our feasibility 344 criterion. In hindsight, our objective to achieve complete follow-up data from 95\% response rate was

345 high - a drop-out rate of more than $20 \%$ is generally considered to compromise the validity of a clinical

346 trial unacceptably (Schulz \& Grimes 2002). Possible reasons for the high drop-out rate may have

347 included participant improvement (such that LBP was no longer a significant concern), or patient

348 perceptions of a lack of relevance of the intervention or the follow-up requests. The protocol for

349 collecting follow-up data via postal questionnaires and SMS reminders was based on the procedure

350 applied in our previous work, which achieved a 4 month follow-up rate of 89\% (Karran et al. 2017b).

351 There were two main differences in the follow-up conditions between these studies. Firstly, our prior

352 study involved participants while they were still waiting for their clinic appointments to be scheduled

353 which may have facilitated engagement, whereas follow-up for the current trial occurred after patients

354 had received their clinic consultation. Secondly, for our initial study, we sent small packets of

355 confectionery with requests for completion and return of questionnaires, whereas in the current trial we

356 did not. Future investigations in this setting should further consider developing an enhanced protocol

357 for achieving follow-up targets and providing participant incentives (Brueton et al. 2013). It may also be

358 important to consider revision of the inclusion criteria to ensure that the patient's problem is of

359 sufficient severity to warrant engagement with the study. Unfortunately, the poor response rate

360 attained in this study impacted the achievement of our secondary objectives. Collected data were used

361 to cautiously inform the determination of sample size for a larger RCT, but was considered insufficient to

362 undertake exploratory analysis of effect.

363

364 The GLITtER intervention was acceptable, of perceived benefit to both patients and clinicians, and was

365 able to be integrated into a standard consultation without significant time burden for clinicians. The 95\%

366 data completion rate on the recommended minimal dataset for LBP research (Deyo et al. 2014) suggests

367 that despite its length, it is not overly onerous for participants. The consent rate of $75 \%$ exceeded our

368 target rate, however it must be acknowledged that a further $8 \%$ of patients who met the primary

369 eligibility criteria did not either confirm or decline consent. Whilst a number of these exclusions were

370 likely to be related to procedural or timing issues it must be also considered that some of these patients 
371 may have opted out of the study (without this action being recorded) - with impact on the overall 372 consent rate.

373

374 This study employed rigorous scientific methods throughout. The protocol was developed in accordance 375 with the SPIRIT checklist and pre-registered on Open Science Framework. The trial was registered on 376 the ANZCTR and has been reported according to the CONSORT statement. Description of the GLITtER 377 intervention followed the TIDieR recommendations for reporting of clinical interventions and the NIHs 378 task force recommended minimum data set for LBP clinical trials was implemented. The specification of 379 feasibility criteria prior to conducting this investigation is a further strength of this study's design. 380 Several weaknesses have also been recognised. There are no standardised criteria for evaluating feasibility, or how achievement of the established criteria should be measured. We used an evidenceinformed but ultimately investigator-led approach to identify and quantify our feasibility criteria, which

384 385 may not have been optimal. Factors such as participant adherence to management recommendations and investigator burden could also have been considered. In addition, we consider that the ability to engage clinicians and administrative personnel is imperative to the success of a clinical trial but we did not formally assess engagement. Our sample size and our follow-up rate were smaller than we expected, which resulted in less data to evaluate our feasibility criteria than we specified as a requirement a priori. The study sample may therefore not be representative of the larger patient population, raising the risk that estimates or interpretations that arise lack precision or are potentially misleading. Last, staff training was not formalised and treatment fidelity was not assessed. While we did calculate an estimated sample size required for a RCT to definitively examine the effect of the GLITtER intervention, this result should be interpreted with caution. Encouragingly, the baseline value for the pain NRS score and the standard deviation of this measure at 3-month follow-up, were very similar to the data recorded in another LBP feasibility trial on which a sample size estimate was also based (Ellard et al. 2017). Based on the assumption that two participants from each SAC would meet final eligibility criteria for inclusion in the study and be randomised, and that clinics operate on 46 weeks of the year - it appears realistic that 92 participants could be recruited annually. Timely achievement of a recruitment target of 126 is likely to be possible, and potential also exists to consider enrolment of participants at 2 or more sites. 
402 This study successfully tested for feasibility of a highly pragmatic approach to addressing the clinical 403 challenge of providing time-efficient, low cost, guideline-informed management of patients with LBP 404 attending secondary care. Based on analysis of our feasibility criteria, a full-scale RCT evaluating the 405 effectiveness of the GLITtER intervention is not recommended without considering strategies that are 406 capable of resolving the recruitment and follow-up issues that we have identified. The shift in clinical 407 pathways raises the possibility that testing of GLITtER in primary care may be indicated. These issues 408 are likely to affect other trials in this population and as such, we humbly suggest that the lessons we 409 have learnt be considered recommendations for the field.

410

\section{Conclusions:}

412 Failure to achieve pre-specified recruitment and follow-up rates was disappointing, however these were 413 important outcomes of a feasibility study. It is realistic to consider that these challenges can be 414 overcome through careful strategy, ample funding and continued partnership with health care 415 providers.

416

417

\section{Acknowledgements}

419 The authors gratefully acknowledge the cooperation and contributions of staff from the Royal Adelaide 420 Hospital Spinal Unit, Spinal Assessment Clinic and Physiotherapy Departments who made this study 421 possible. We also express our thanks to the patients involved.

422

423

424 References

425

426

427

(ABS). 2015. AIHW analysis of unpublished ABS Australian Health Survey, 2014-2015. Available at

428 www.aihw.gov.au/back-problems/prevalence/\#source-table5

429

430

(NICE) NIFHACE. 2016. Low Back Pain and Management in over 16s: Assessment and Management. Available at https://www.nice.org.uk/quidance/ng59/chapter/Recommendations. (Viewed 25 July, 2017) 
431 Brinjikji W, Luetmer P, Comstock B, Bresnahan B, Chen L, Deyo R, Halabi S, Turner J, Avins A, and James

432

433

434

435

436

437

438

439

440

441

442

443

444

445

446

447

448

449

450

451

452

453

454

455

456

457

458

459

460

461

K. 2014. Systematic Literature Review of Imaging Features of Spinal Degeneration in Asymptomatic Populations. American Journal of Neuroradiology 36:811-816.

Brueton VC, Tierney J, Stenning S, Harding S, Meredith S, Nazareth I, and Rait G. 2013. Strategies to improve retention in randomised trials. The Cochrane Library Issue 12. Art. No.: MR000032. DOI: 10.1002/14651858.MR000032.pub2.

Carragee E, Alamin T, Cheng I, Franklin T, van den Haak E, and Hurwitz E. 2006. Are first-time episodes of serious LBP associated with new MRI findings? The Spine Journal 6:624-635.

Carragee EJ, Alamin TF, Miller JL, and Carragee JM. 2005. Discographic, MRI and psychosocial determinants of low back pain disability and remission: a prospective study in subjects with benign persistent back pain. The Spine Journal 5:24-35.

Coulter A. 1998. Managing demand: Managing demand at the interface between primary and secondary care. BMJ: British Medical Journal 316:1974.

De Schepper EI, Koes BW, Oei EH, Bierma-Zeinstra SM, and Luijsterburg PA. 2016. The added prognostic value of MRI findings for recovery in patients with low back pain in primary care: a 1-year follow-up cohort study. European Spine Journal 25:1234-1241.

Deyo RA, Dworkin SF, Amtmann D, Andersson G, Borenstein D, Carragee E, Carrino J, Chou R, Cook K, DeLitto A, Goertz C, Khalsa P, Loeser J, Mackey S, Panagis J, Rainville J, Tosteson T, Turk D, Von Korff M, and Weiner DK. 2014. Report of the NIH Task Force on Research Standards for Chronic Low Back Pain. The Journal of Pain 15:569-585. http://dx.doi.org/10.1016/j.jpain.2014.03.005

Ellard DR, Martin Underwood, Felix Achana, James HL Antrobus, Shyam Balasubramanian, Sally Brown, Melinda Cairns, James Griffin, Frances Griffiths, Kirstie Haywood, Charles Hutchinson, Ranjit Lall, Stavros Petrou, Nigel Stallard, Colin Tysall, David A Walsh, and Sandhu H. 2017. Facet joint injections for people with persistent non-specific low back pain (Facet Injection Study): a feasibility study for a randomised controlled trial. HEALTH TECHNOLOGY ASSESSMENT, NIH (National Institute for Health Research) 21. DOI 10.3310/hta21300

Graves JM, Fulton-Kehoe D, Jarvik JG, and Franklin GM. 2012. Early imaging for acute low back pain: one-year health and disability outcomes among Washington State workers. Spine 37:1617-1627. Hasenbring MI, and Pincus T. 2015. Effective reassurance in primary care of low back pain: what messages from clinicians are most beneficial at early stages? Clinical Journal of Pain 31:133-136. 
462 463

464

465

466

467

468

469

470

471

472

473

474

475

476

477

478

479

480

481

482

483

484

485

486

487

488

489

490

491

492

493

Hertzog MA. 2008. Considerations in determining sample size for pilot studies. Research in Nursing \& Health 31:180-191.

Jensen OK, Nielsen CV, Sorensen JS, and Stengaard-Pedersen K. 2014. Type 1 Modic changes was a significant risk factor for 1-year outcome in sick-listed low back pain patients: a nested cohort study using magnetic resonance imaging of the lumbar spine. Spine Journal: Official Journal of the North American Spine Society 14:2568-2581.

Karran E, Yau Y, Hillier S, and Moseley GL. 2017. The reassuring potential of spinal imaging results: Development and testing of a brief, psycho-education intervention for patients attending secondary care. European Spine Journal. 10.1007/s00586-017-5389-8

Karran EL, McAuley JH, Traeger AC, Hillier SL, Grabherr L, Russek LN, and Moseley GL. 2017. Can screening instruments accurately determine poor outcome risk in adults with recent onset low back pain? A systematic review and meta-analysis. BMC Medicine 15:13. https://doi.org/10.1186/s12916-016-0774-4

Karran EL, Traeger AC, McAuley JH, Hillier SL, Yau Y-H, and Moseley GL. 2017. The value of prognostic screening for patients with low back pain in secondary care. The Journal of Pain 18:673-686.

Koes BW, van Tulder M, Lin C-WC, Macedo LG, McAuley J, and Maher C. 2010. An updated overview of clinical guidelines for the management of non-specific low back pain in primary care. European Spine Journal 19:2075-2094.

Li A, and Yen D. 2010. Changes in referral pattern to a surgeon for low back pain: 1996 versus 2009. Healthcare quarterly (Toronto, Ont) 13:91-95.

Mors $\varnothing$ L, Kent P, Albert HB, and Manniche C. 2013. Is the psychosocial profile of people with low back pain seeking care in Danish primary care different from those in secondary care? Manual therapy 18:54-59.

Mors $\varnothing$ L, Kent P, Manniche C, and Albert HB. 2014. The predictive ability of the STarT Back Screening Tool in a Danish secondary care setting. European Spine Journal 23:120-128.

O'Connell NE, Cook CE, Wand BM, and Ward SP. 2016. Clinical guidelines for low back pain: A critical review of consensus and inconsistencies across three major guidelines. Best Practice \& Research Clinical Rheumatology 30:968-980.

Pincus T, and McCracken LM. 2013. Psychological factors and treatment opportunities in low back pain. Best Practice \& Research Clinical Rheumatology 27:625-635.

Qaseem A, Wilt TJ, McLean RM, and Forciea MA. 2017. Noninvasive Treatments for Acute, Subacute, and Chronic Low Back Pain: A Clinical Practice Guideline From the American College of 
494

495

496

497

498

499

500

501

502

503

504

505

506

507

508

509

510

511

512

513

514

515

516

517

518

519

520

521

522

523

524

PhysiciansNoninvasive Treatments for Acute, Subacute, and Chronic Low Back Pain. Annals of Internal Medicine 166:514-530.

Robarts S, Stratford P, Kennedy D, Malcolm B, and Finkelstein J. 2017. Evaluation of an advancedpractice physiotherapist in triaging patients with lumbar spine pain: surgeon-physiotherapist level of agreement and patient satisfaction. Canadian Journal of Surgery 60:266-271.

Roland M, and Morris R. 1983. A study of the natural history of back pain: part I: development of a reliable and sensitive measure of disability in low-back pain. Spine 8:141-144.

Roland M, and van Tulder M. 1998. Should radiologists change the way they report plain radiography of the spine? The Lancet 352:229-230.

Schulz KF, and Grimes DA. 2002. Sample size slippages in randomised trials: exclusions and the lost and wayward. The Lancet 359:781-785.

Sears ED, Caverly TJ, Kullgren JT, Fagerlin A, Zikmund-Fisher BJ, Prenovost K, and Kerr EA. 2016.

Clinicians' perceptions of barriers to avoiding inappropriate imaging for low back pain-knowing is not enough. JAMA Internal Medicine 176:1866-1868. 10.1001/jamainternmed.2016.6364

Steffens D, Hancock MJ, Maher CG, Williams C, Jensen TS, and Latimer J. 2014. Does magnetic resonance imaging predict future low back pain? A systematic review. European Journal of Pain 18:755765.

Stochkendahl MJ, Kjaer P, Hartvigsen J, Kongsted A, Aaboe J, Andersen M, Andersen M $\varnothing$, Fournier G, Højgaard B, and Jensen MB. 2017. National Clinical Guidelines for non-surgical treatment of patients with recent onset low back pain or lumbar radiculopathy. European Spine Journal https://doi.org/10.1007/s00586-017-5099-2.

Suri P, Boyko EJ, Goldberg J, Forsberg CW, and Jarvik JG. 2014. Longitudinal associations between incident lumbar spine MRI findings and chronic low back pain or radicular symptoms: retrospective analysis of data from the longitudinal assessment of imaging and disability of the back (LAIDBACK). BMC Musculoskeletal Disorders 15:152. http://www.biomedcentral.com/1471-2474/15/152

Thabane L, Ma J, Chu R, Cheng J, Ismaila A, Rios LP, Robson R, Thabane M, and Goldsmith CH. 2010. A tutorial on pilot studies: The what, why and How. BMC Medical Research Methodology 10. $10.1186 / 1471-2288-10-1$

Tkachuk GA, and Harris CA. 2012. Psychometric Properties of the Tampa Scale for Kinesiophobia-11 (TSK-11). The Journal of Pain 13:970-977. http://dx.doi.org/10.1016/j.jpain.2012.07.001 
525 Traeger AC, Hübscher M, Henschke N, Moseley GL, Lee H, and McAuley JH. 2015. Effect of Primary Care-

526

527

528

529

530

531

532

533

534

535

536

537

538

539

540

541

542 Based Education on Reassurance in Patients With Acute Low Back Pain: Systematic Review and Meta-analysis. JAMA Internal Medicine 175:733-743.

Traeger AC, O'Hagan ET, Cashin A, and McAuley JH. 2017. Reassurance for patients with non-specific conditions-a user's guide. Brazilian Journal of Physical Therapy 21:1-6.

Vos T, Allen C, Arora M, Barber RM, Bhutta ZA, Brown A, Carter A, Casey DC, Charlson FJ, and Chen AZ. 2016. Global, regional, and national incidence, prevalence, and years lived with disability for 310 diseases and injuries, 1990-2015: a systematic analysis for the Global Burden of Disease Study 2015. The Lancet 388:1545.

Webster BS, and Cifuentes M. 2010. Relationship of early magnetic resonance imaging for work-related acute low back pain with disability and medical utilization outcomes. Journal of Occupational and Environmental Medicine 52:900-907.

Wong JJ, Côté P, Sutton DA, Randhawa K, Yu H, Varatharajan S, Goldgrub R, Nordin M, Gross DP, Shearer HM, Carroll L, Stern PJ, Ameis A, Southerst D, Mior S, Stupar M, Varatharajan T, and TaylorVaisey A. 2017. Clinical practice guidelines for the noninvasive management of low back pain: A systematic review by the Ontario Protocol for Traffic Injury Management (OPTIMa)

Collaboration. European Journal of Pain 21:201-216. 10.1002/ejp.931 


\section{Table $\mathbf{1}$ (on next page)}

Description of the intervention and control consultations (consistent with the TIDieR reporting checklist (see www.equator-network.org )) 
WHY Goal: To supplement and enhance the standard SAC consultation with a strategy designed to optimise patient reassurance and facilitate

engagement in an active recovery.

\section{Materials:}

- GLITtER Framework used for clinicians training (see Appendix 3)

- Visual aid A (used during intervention delivery): graph of prevalence of degenerative features in asymptomatic adults (see Appendix 7)

- Take-home information resource (see Appendix 8). This was designed as a series of 4 posters to be displayed one week at a time.

- Links to online information (incorporated into take-home resource and delivered via smartphone text messages):

- The truth about back pain

(https://www.youtube.com/watch?v=b-cBtPSfOHc)

- Tame the Beast

(https://www.youtube.com/watch?v=XwBYkw-iZdQ)

- How to start exercising and stick to it

(https://www.helpguide.org/articles/healthy-living/how-to-start-

exercising-and-stick-to-it.htm)

- Understanding pain in less than 5 minutes

(https://www.youtube.com/embed/qEWc2XtaNwg)

- $\quad$ Letter to General Practitioner (see Appendix 9)

\section{Procedures:}

- All procedures implemented in the standard consultation were included in the GLITtER consultation.

- Additional procedures (unique to the GLITtER consultation):

i. Provide detailed information about 'normal', age- relevant imaging findings and involve visual aid $A$.

ii. In addition to explaining patient's imaging findings, explain that:

Scans (on their own) do not explain much about:

- Your current pain (e.g. why you have good days and bad)

- The activity you are capable of, or

- How likely you are to recover (because the changes on your scans will still be there when your pain goes away)

iii. Re-interpret imaging findings, highlighting 'positive' features. E.g.

- Demonstrate spinal features that offer structural stability and emphasise the inherent strength of the spine.

- Demonstrate musculature and joints - structures that need movement to be optimally healthy

iv. Promote using the 'TICK list' as a strategy for increasing planned activity/exercise (see Appendix 8)
Goal: To provide comprehensive assessment and management of patients with LBP attending the SAC. Key objectives are to identify potential surgical candidates and to transfer the care of non-surgical candidates to General Practitioner supported community based care.[1]

\section{Materials:}

- No physical or informational materials are routinely used or provided

\section{Procedures:}

A standard consultation involves:

- Subjective examination/patient history

- Physical examination, including neurological assessment

- Review of imaging and relevant investigations

- Discussion of relevant findings (from examination and investigations)

- Discussion of management recommendations

- Written correspondence with the patients' General Practitioner 
Abbreviations: GLITtER, Green Light Imaging interpretation To Enhance Recovery; LBP, low back pain; SAC, Spinal Assessment Clinic; SMS, short message service;

* Details of training provided to Clinicians 1 and 2 is provided in the manuscript.

1. Beard M, Orlando JF, Kumar S (2016) Overcoming the tyranny of distance: An audit of process and outcomes from a pilot telehealth spinal assessment clinic. Journal of Telemedicine and Telecare: 1357633X16664851 
Table 2 (on next page)

Feasibility considerations, criteria and outcomes 


\begin{tabular}{lll}
\hline $\begin{array}{l}\text { Feasibility } \\
\text { considerations }\end{array}$ & Criteria & Outcome \\
\hline Process & $\begin{array}{l}\text { One participant per clinician per (weekly) clinic can be } \\
\text { Recruitment rate }\end{array}$ & Review \\
recruited (4 participants recruited at each clinic) \\
Follow-up rate & $\begin{array}{l}\text { At least 70\% of all eligible patients can be recruited } \\
\text { Complete follow-up data can be obtained for at least 95\% of } \\
\text { participants }\end{array}$ & Review \\
\hline
\end{tabular}

\section{Resource}

Data collection:

Participant burden*

95\% completion of baseline data (Recommended Minimum Review Dataset for LBP Research and TSK-11) prior to SAC appointment

Clinician/SAC

burden

GLITtER consultations do not extend clinic appointments by

Achieved more than 10 minutes (on average)

Acceptability
Patient
acceptability
Clinician
acceptability \&
perceived benefit

$>80 \%$ of responses to questions 1-7 of the Participant

Achieved

Experience Questionnaire rated as "agree" or "strongly agree"

SAC Clinicians (delivering the GLITtER intervention) report that they are "confident" or "very confident" when asked:

- "How confident are you that you could integrate GLITtER

Achieved acceptability \& into standard practice on an ongoing basis?" and

- $\quad$ "How confident are you that integrating GLITtER would enhance SAC care?" (4-point scale)

\section{Scientific}

Determination of

Use the standard deviation of the NRS score for pain at 3-

Achieved

sample size for month follow-up to calculate sample size

appropriately

powered RCT

Exploratory

Calculate between-group differences for changes in pain,

Achieved

analyses of effect disability, and kinaesiophobia (from baseline to 3 month follow-up).

Identify ceiling or floor effects

Presence or absence of ceiling or floor effect identified and quantified

Achieved

${ }^{*}$ criterion not pre-specified in protocol

Abbreviations: LBP, low back pain; TSK, Tampa Scale for Kinaesiophobia; SAC, Spinal Assessment Clinic; RCT, randomised controlled trial 
Table 3 (on next page)

Key baseline participant characteristics 


\begin{tabular}{|c|c|c|c|}
\hline & \multirow{2}{*}{$\begin{array}{l}\text { Total Sample } \\
(\mathrm{N}=31)\end{array}$} & \multicolumn{2}{|l|}{ Group } \\
\hline & & $\begin{array}{l}\text { GLITtER } \\
\text { Consultation } \\
(\mathrm{N}=20)\end{array}$ & $\begin{array}{l}\text { Standard } \\
\text { Consultation } \\
(\mathrm{N}=11)\end{array}$ \\
\hline Age (mean, SD) & $50.1(14.0)$ & $52.4(11.7)$ & $46.1(17.4)$ \\
\hline Gender (\% female) & $63 \%$ & $65 \%$ & $55 \%$ \\
\hline $\begin{array}{l}\text { Pain NRS (mean, SD) } \\
\text { In the past } 7 \text { days, how would you rate } \\
\text { the intensity of your pain on average? } \\
\text { (Question } 3^{*} \text { ) }\end{array}$ & $\begin{array}{l}6.3(1.8) \\
\text { (97\% complete } \\
\text { responses) }\end{array}$ & $\begin{array}{l}6.2(1.8) \\
\text { ( } 95 \% \text { complete } \\
\text { responses) }\end{array}$ & $\begin{array}{l}6.5(2.0) \\
\text { (100\% complete } \\
\text { responses) }\end{array}$ \\
\hline $\begin{array}{l}\text { Disability NRS (mean, SD) } \\
\text { In the past } 7 \text { days, how much has pain } \\
\text { interfered with your day-to-day } \\
\text { activities? (Question } 7 * \text { ) }\end{array}$ & $\begin{array}{l}5.8(2.1) \\
\text { (100\% complete } \\
\text { responses) }\end{array}$ & $\begin{array}{l}5.9(1.8) \\
\text { (100\% complete } \\
\text { responses) }\end{array}$ & $\begin{array}{l}5.6(2.5) \\
(100 \% \text { complete } \\
\text { responses) }\end{array}$ \\
\hline $\begin{array}{l}\text { Pain Catastrophising Scale (mean, } \\
\text { SD) }\end{array}$ & $\begin{array}{l}38.9(7.3) \\
\text { (74\% complete } \\
\text { responses) }\end{array}$ & $\begin{array}{l}40.5(7.1) \\
\text { (65\% complete } \\
\text { responses) }\end{array}$ & $\begin{array}{l}36.7(7.3) \\
\text { (91\% complete } \\
\text { responses) }\end{array}$ \\
\hline
\end{tabular}

Abbreviations: SD, standard deviation; NRS, numeric rating scale

*From Recommended Minimum Dataset for Low Back Pain Research, see eSupplement 6 


\section{Table 4(on next page)}

Baseline, 3 month follow-up and change scores for pain, disability and kinaesiophobia 


\begin{tabular}{|c|c|c|c|c|}
\hline & \multicolumn{2}{|l|}{ Baseline scores } & \multicolumn{2}{|c|}{3 month follow-up } \\
\hline & $\begin{array}{l}\text { GLITtER } \\
\text { Mean (SD), N }\end{array}$ & $\begin{array}{l}\text { Standard } \\
\text { Mean (SD), N }\end{array}$ & $\begin{array}{l}\text { GLITtER } \\
\text { Mean (SD), N }\end{array}$ & $\begin{array}{l}\text { Standard } \\
\text { Mean (SD), N }\end{array}$ \\
\hline $\begin{array}{l}\text { Pain } \\
\text { (NRS) }\end{array}$ & $\begin{array}{l}6.2(1.8) \\
N=19\end{array}$ & $\begin{array}{l}6.5(2.0) \\
\mathrm{N}=7\end{array}$ & $\begin{array}{l}6.5(2.0) \\
N=11\end{array}$ & $\begin{array}{l}5.3(2.1) \\
\mathrm{N}=6\end{array}$ \\
\hline $\begin{array}{l}\text { Disability } \\
\text { (NRS) }\end{array}$ & $\begin{array}{l}5.9(1.8) \\
\mathrm{N}=20\end{array}$ & $\begin{array}{l}4.2(2.8) \\
N=7\end{array}$ & $\begin{array}{l}5.6(2.5) \\
\mathrm{N}=11\end{array}$ & $\begin{array}{l}4.2(2.9) \\
\mathrm{N}=6\end{array}$ \\
\hline $\begin{array}{l}\text { Kinaesio- } \\
\text { phobia } \\
\text { (TSK-11) }\end{array}$ & $\begin{array}{l}40.6(7.1) \\
N=13\end{array}$ & $\begin{array}{l}38.9(7.2) \\
N=5\end{array}$ & $\begin{array}{l}36.7(7.3) \\
N=10\end{array}$ & $\begin{array}{l}30.0(7.8) \\
\mathrm{N}=5\end{array}$ \\
\hline
\end{tabular}

Abbreviations: SD, standard deviation; NRS, numeric rating scale; TSK, Tampa Scale for Kinaesiophobia 


\section{Figure 1}

StudyFlow: Prospective, comparative, feasibility trial of GLITTER

Abbreviations: TSK, Tampa Scale for Kinaesiophobia; SMS, short message service; NRS, numeric rating scale; RMDQ, Roland Morris Disability Questionnaire 
Clinic lists screened 2-3/52 prior to clinic. Patients meeting initial eligibility criteria mailed a 'Research Notification' letter to offer opportunity to opt- out of research involvement

Patient referrals and case-notes screened immediately prior to clinic to confirm patient suitability for study inclusion

\begin{tabular}{|c|}
\hline $\begin{array}{c}\text { Potential participants approached in waiting room and eligibility } \\
\text { confirmed. Study information provided. }\end{array}$ \\
\hline $\begin{array}{c}\text { Patients agreeing to be involved completed signed consent and } \\
\text { baseline questionnaires (recommended minimum dataset for low } \\
\text { back pain research, TSK) }\end{array}$ \\
\hline $\begin{array}{c}\text { Pragmatic allocation of potential participants to SAC clinicians } \\
\text { (based on patient attendance order and clinician availability) }\end{array}$ \\
\hline
\end{tabular}
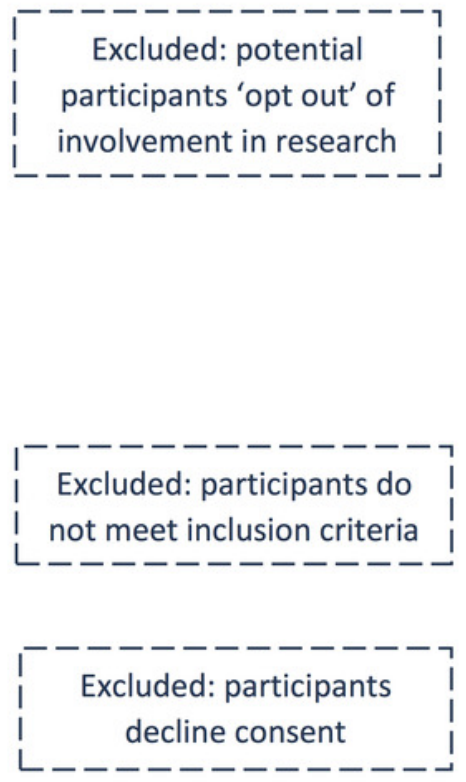

\section{Patient attends clinic appointment with SV or CC (Clinicians 1 \&2)}

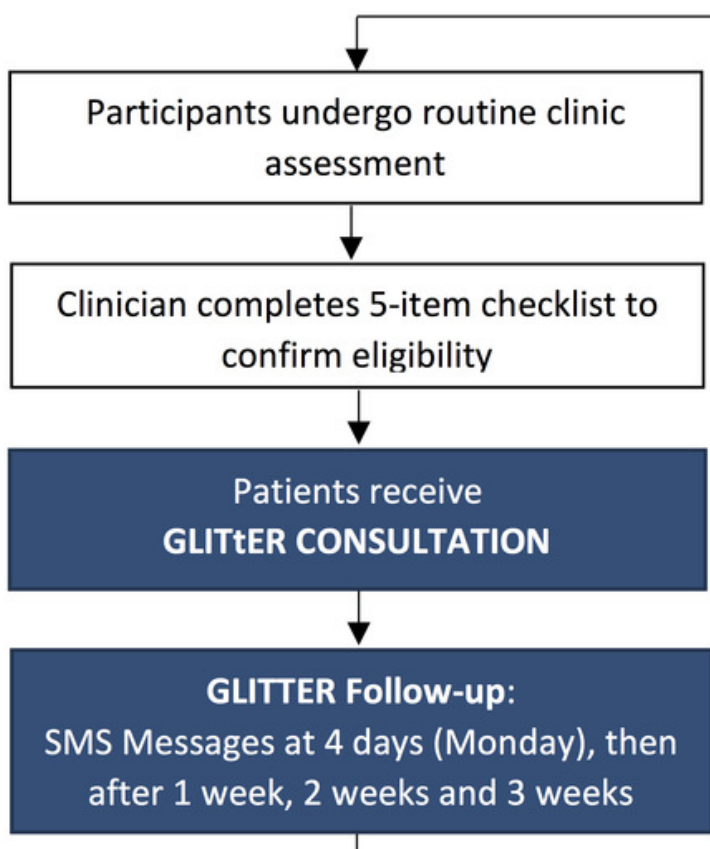

Patient attends clinic appointment with AW or MJ (Clinicians 3 \& 4)

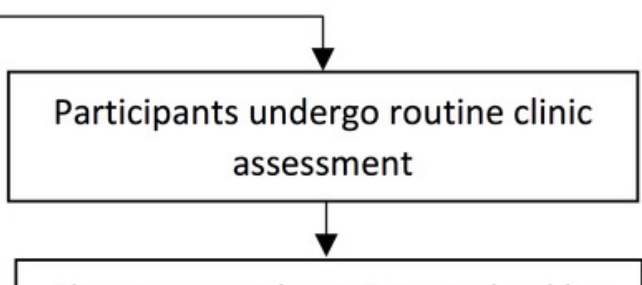

Clinician completes 5 -item checklist to confirm eligibility

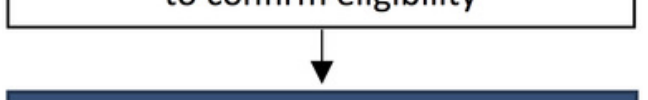

Patients receive

STANDARD CONSULTATION

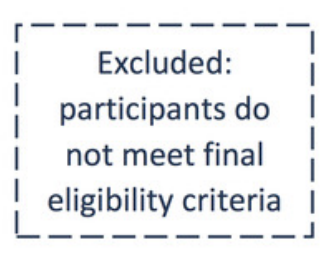

1 month follow-up: Pain NRS, Disability NRS, RMDQ, Reassurance Questions, Healthcare Utilisation, TSK, Evaluation/Satisfaction Questions*

3 month follow-up: Pain NRS, Disability NRS, RMDQ, Healthcare Utilisation, TSK 
Figure 2

\section{CONSORT flow diagram}

Screening:

PRELIMINARY

Screening:

PRIMARY

Enrolment

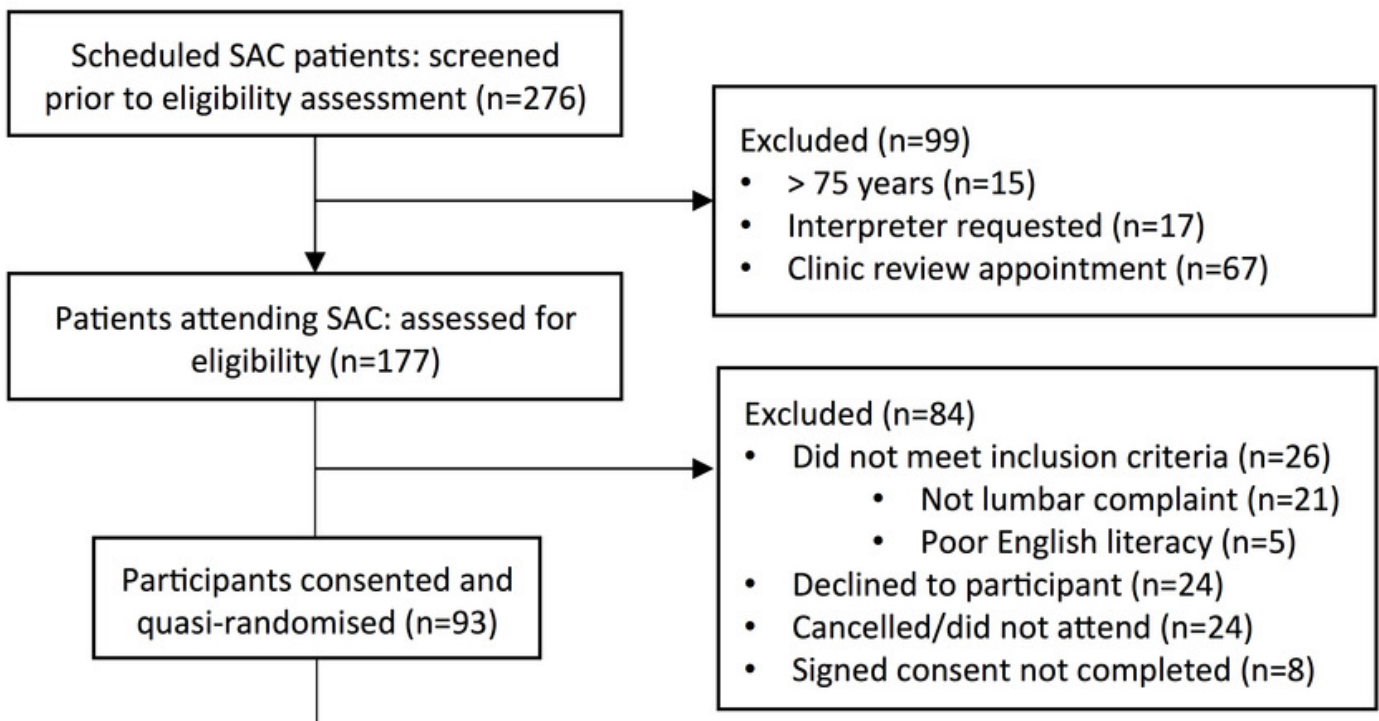

\section{Screening: \\ FINAL}

Participants meeting final eligibility criteria (as assessed by clinicians) and enrolled in study ( $n=31)$
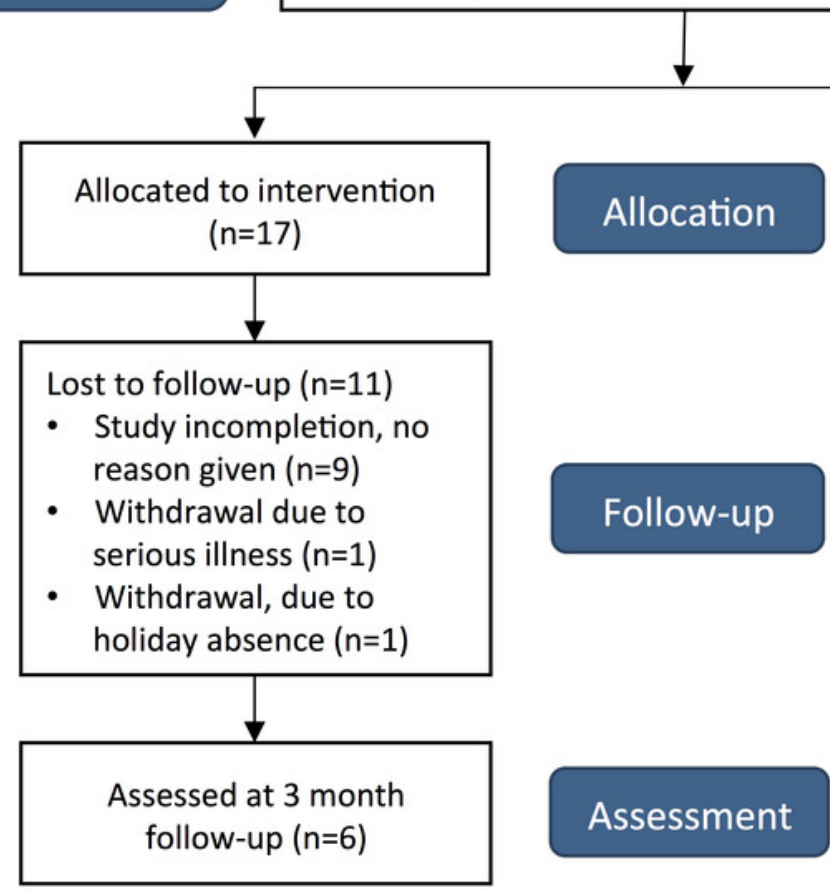

Allocated to control $(n=14)$

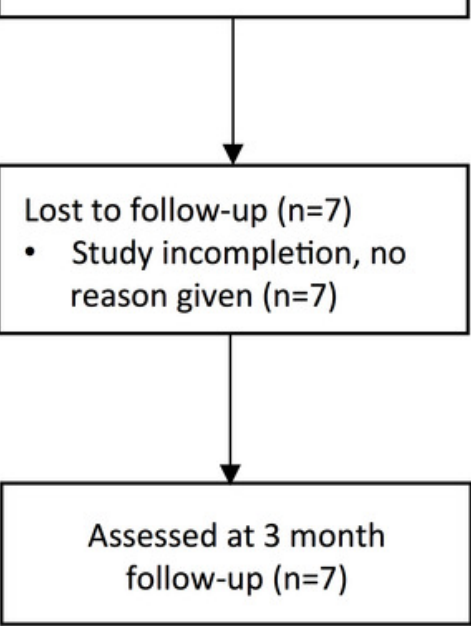

